\title{
Exploration of Long-term CD4 Profile in HIV Patients Under HAART at Mizan-Tepi University Teaching Hospital and Tepi General Hospital, South Western Ethiopia
}

\author{
Solomon Abebaw Andargie*, Assaye Belay Gelaw \\ Department of Statistics, Mizan-Tepi University, Tepi, Ethiopia \\ Email address: \\ solabew@gmail.com (S. A. Andargie) \\ ${ }^{*}$ Corresponding author \\ To cite this article: \\ Solomon Abebaw Andargie, Assaye Belay Gelaw. Exploration of Long-term CD4 Profile in HIV Patients Under HAART at Mizan-Tepi \\ University Teaching Hospital and Tepi General Hospital, South Western Ethiopia. Pure and Applied Mathematics Journal. \\ Vol. 9, No. 5, 2020, pp. 91-95. doi: 10.11648/j.pamj.20200905.12
}

Received: August 18, 2020; Accepted: August 27, 2020; Published: September 14, 2020

\begin{abstract}
Currently, because of the wide availability and free service of HAART, HIV/AIDS related morbidity and mortality has decreased significantly. However, patients accessing antiretroviral treatment (ART) programmes in sub-Saharan Africa frequently have very advanced immunodeficiency and various reserches suggest that such patients may have diminished capacity for CD4 cell count recovery. The objective of this study was to investigate the long-term effect of highly active antiretroviral therapy on the CD4 lymphocyte count of HIV-infected Patients. Subjects from the multicenter HAART Program cohort (from Mizan-Tepi University Teaching Hospital and Tepi General Hospital), aged 18 years or older and had an ART treatment start date in between February 1, 2017 to January 31, 2019 were enrolled in the present study and followed for a maximum of 3 years. Liner mixed model with nested random effect were used to model the longitudianl CD4 count over time. The data reveal robust CD4 responses to ART that are continual over several years. Being under HAART for long period and having baseline CD4 count greater than 150 were positively associated with CD4 increment over time while starting ART at late stage (Stage 3 or 4 ) and being male are negatively assocted with CD4 increment over time. These study show strong and repetitive CD4 response to ART among patients remaining on therapy. Earlier HIV diagnosis and initiation of ART could significantly progress patient outcomes in the study area.
\end{abstract}

Keywords: CD4counts, HAART, ART, Liner Mixed Model, Patients

\section{Introduction}

AIDS is a disease in which the immune system of a body becomes weak and unable to fight against opportunistic infections and other illness which take the advantage of the weaken immune system. Once the person is infected with HIV, the virus enters the body and grows up to the stage where it damages or kills the white blood cells weakening the immune system and leaving the person vulnerable to various opportunistic infections. At global level, the number of people living with AIDS at the end of 2017 was estimated to be more than 34 million, with 2.5 million new infections and 1.7 million deaths. More than two-thirds (69 percent) of all people living with HIV, 23.5 million, live in sub-Saharan Africa-including 91 percent of the world's HIV-positive children. In 2011, an estimated 1.8 million people in the region became newly infected. An estimated 1.2 million adults and children died of AIDS, accounting for 71 percent of the world's AIDS deaths in 2011. This infection still requires new discovery. Particularly, the epidemic still remains high in Sub-Saharan Africa indicating that this region is still the most severely affected region $[1,3,4,7,8]$.

The emergency of HAART have shown a dramatic change in control the burden of HIV/AIDS. Various studies from the developed as well as developing countries reported robust improvements in CD4 cell counts following ART initiation. CD4 cell count and HIV viral load in response to antiretroviral therapy (ART) are important measures of the efficacy of ART in individual patients and of the effectiveness of ART in populations of patients enrolled in 
HIV care and treatment programs [9, 10].

However, few data exist on long-term CD4 response to ART among patients receiving care, where HIV RNA testing is not generally available or conducted. Several studies in Europe and North America have reported robust improvements in CD4 cell counts following ART initiation in clinical trials and in observational studies [5, 11]. Although data from resourcelimited settings are less commonly available, some investigators of research and scale-up cohorts in sub-Saharan Africa, Barbados, Brazil and Cambodia have reported effects of ART on clinical and immunologic outcomes that were comparable to those observed in resource-rich settings. However the majority of the studies in developing countries have had follow-up times of 1 to 2 years, Thus while it has been shown in developed countries the degree which CD4 responses can be maintained for longer periods of time after ART initiation in developing countries has not been demonstrated $[6,7,12]$. The study was conducted to describe the determinants of CD4 response trajectories up to 3 years after initiation of ART in Mizan-Tepi University Teaching Hospital and Tepi General Hospital, South Western Ethiopia.

\section{Methods}

\subsection{Study Area and Design}

The HAART data used in this study was obtained from Mizan-Tepi University Teaching Hospital and Tepi General Hospital HIV/AIDS clinic, South Western Ethiopia. The hospital gives VCT, PMTCT and free ART services according to Ethiopian National Guideline among others (FMoH, 2007) for people living in Mizan-aman Town and Tepi Town respectively. Patients start ART after they checked for medical eligibility and ready (counseled for adherence) for ART. Patients become eligible for HAART with either a documented CD4 count less than 200 cells $/ \mathrm{mm} 3$ or if they are classifed as
WHO stage 4. However, CD4 threshold for initiation was changed from 200 to 350 cells $/ \mathrm{mm} 3$ in 2012 . The treating physician is responsible in prescribing the appropriate initial HAART regimen. The hospital collects demographic, laboratory (such as CD4), and clinical data at baseline. All patients initiating ART who were ART naive, aged 18 years or older and had an ART treatment start date in between February 1, 2017 to January 31, 2019 were eligible for this study.

\subsection{Statistical Analysis}

For immunological outcome analysis CD4 counts was used as a primary indictor. The longitudinal response, the number of CD4 counts per mm3 of blood, was measured at irregular time interval even if it is expected to be measured in every 6 months. A CD4 counts at the start of treatment was considered as the CD4 measurement at time zero (baseline) and the time for subsequent CD4 measurements was calculated in month from the start of treatment. Patients were classified as being dead, lost to program, or presumed to be alive and on ART based on all available information at the time of the closure of the study database. Lost to follow-up was defined as not returning to clinic for 1 year or longer after the last recorded visit. Time on ART was measured from the ART initiation date to the last known encounter. Data were censored at the closing date of each cohort out to a maximum of 3 years post-ART-initiation. Descriptive analyses were used to compare baseline characteristics study subjects.

Linear mixed regression models were used to evaluate the association between independent variables and CD4 response over time on ART. We constructed a multi-level model (CD4 measurements within patients nested within study sites) with random intercepts and slope for each patient and Study site. CD4 count response values were log transformed to achieve a normal distribution. The proposed model can be specified as follow; [13]

$$
Y_{i j k}=\beta_{0}+\beta_{1} t_{i j k}+\beta_{2} t_{i j k}^{2}+\beta^{t} X+b_{0 k}+b_{0 i(k)}+b_{1 k} t_{i j}+b_{1 i(k)} t_{i j}+\varepsilon_{i j k}
$$

where, $Y_{i j k}$ is the log transformed CD4 count measured at time $t_{i j k}$ for the $j^{\text {th }}$ subject from $k^{\text {th }}$ health center. $\beta_{0}, \beta_{1}$, and $\beta_{2}$ are the population intercept, time effect and squared time effect, respectively. $X$ a $N x p$ design matrix for candidate covariates and the vector $\beta$ is there associated parameter. The following variables were considered for their influence on CD4 trajectory: gender, age at ART initiation, baseline CD4 count, clinical stage of disease, and initial ART regimen. Further, we introduce a vector of random effect $b^{t}=\left(b_{0 k}, b_{0 i(k)}, b_{1 k}, b_{1 i(k)}\right)$ to represent individual deviation from the population average, where, $b_{0 k}$ and $b_{0 i(k)}$ are random intercept for the $k^{\text {th }}$ health center and for the $j^{\text {th }}$ subject nested within the $k^{t h}$ health center and $b_{1 k}$ and $b_{1 i(k)}$ random slopes for the $k^{\text {th }}$ health center and for the $j^{t h}$ subject nested within the $k^{t h}$ health center. We assumed the health center and subject nested within health center random structure to follow a multivariate normal distribution with mean vector 0 and variance-covariance,

$$
\Sigma=\left[\begin{array}{cccc}
\delta_{b_{o k}}^{2} & \delta_{b_{o k, b_{1 k}}} & 0 & 0 \\
\delta_{b_{o k, b_{1 k}}} & \delta_{b_{1 k}}^{2} & \delta_{b_{o j(k)}}^{2} & \delta_{b_{o j(k), b_{1 j(k)}}} \\
0 & 0 & \delta_{b_{o j(k), b_{1 j(k)}}} & \delta_{b_{1 j(k)}}^{2}
\end{array}\right]
$$

At last, the residuals, $\varepsilon_{i j k}$, are assumed to follow independently and identically distributed normall $N\left(0, \delta_{\varepsilon}^{2}\right)$. Fixed effect part of the model parameters are estimated using maximum likelihood (ML) and restricted maximum likelihood (REML) was used for the variance components. Statistical significance of the random effects was tested by using an approximate likelihood ratio test [14].

\section{Results}

Four hundred seventy six patients, 240 from Mizan -Tepi Teaching Hospital and 236 from Tepi General Hospital, were included in the study. The majority of these patients were female $(62.58 \%)$ with a median age of 30 years. The median CD4 count 
at initiation was 121.15 and majority of the patients were at WHO stage 1 and 3 . The most common regimens initiated were treatment $\mathrm{d} 4 \mathrm{~T}+3 \mathrm{TC}+\mathrm{NVP}$ and $\mathrm{TDF}+3 \mathrm{TC}+\mathrm{EFV}$.

Table 1. Base line characteristics of study subjects.

\begin{tabular}{llll}
\hline Sex & All (\%) & Mizan (\%) & Tepi (\%) \\
\hline Female & $303(62.58)$ & $141(58.1)$ & $162(67.47)$ \\
Male & $173(37.42)$ & $98(41.89)$ & $75(32.53)$ \\
WHO stage & & & \\
1 & $157(33.27)$ & $55(22.92)$ & $102(43.7)$ \\
2 & $147(30.48)$ & $72(29.64)$ & $75(31.32)$ \\
3 & $153(31.67)$ & $102(41.5)$ & $51(21.68)$ \\
4 & $19(4.57)$ & $12(5.93)$ & $6(3.2)$ \\
Treatment & & & \\
d4T+3TC+NVP & $126(26.29)$ & $22(9.88)$ & $104(42.96)$ \\
d4T+3TC+EFV & $68(14.54)$ & $30(13.83)$ & $38(15.3)$ \\
AZT+3TC+NVP & $93(19.52)$ & $87(35.57)$ & $6(3.2)$ \\
AZT+3TC+EFV & $76(16.13)$ & $57(23.71)$ & $19(8.43)$ \\
TDF+3TC+EFV & $110(22.9)$ & $40(16.6)$ & $70(29.31)$ \\
TDF+3TC+NVP & $3(0.8)$ & $1(0.4)$ & $2(0.6)$ \\
Age (Median) & $31(10.75)$ & $31(9)$ & $30(11)$ \\
CD4 (Median) & $152.5(121.15)$ & $171(124)$ & $159(125)$ \\
\hline
\end{tabular}

Figure 1 shows the individual CD4 profile and smoothed average profile over time on ART stratified by sex, clinical stage, initiation regimen, baseline CD4, and age of the patient. Increases were steeper in females than males, with differences widening with time on ART. The CD4 profile overtime for clinical stages, NVP versus EFV, TDF versus d4T or AZT looks similar. The study also found that, increases were steeper in patients who start treatment while their CD4 count was above 150 than those who start with CD4 count below 150, with differences narrowing with time on ART.
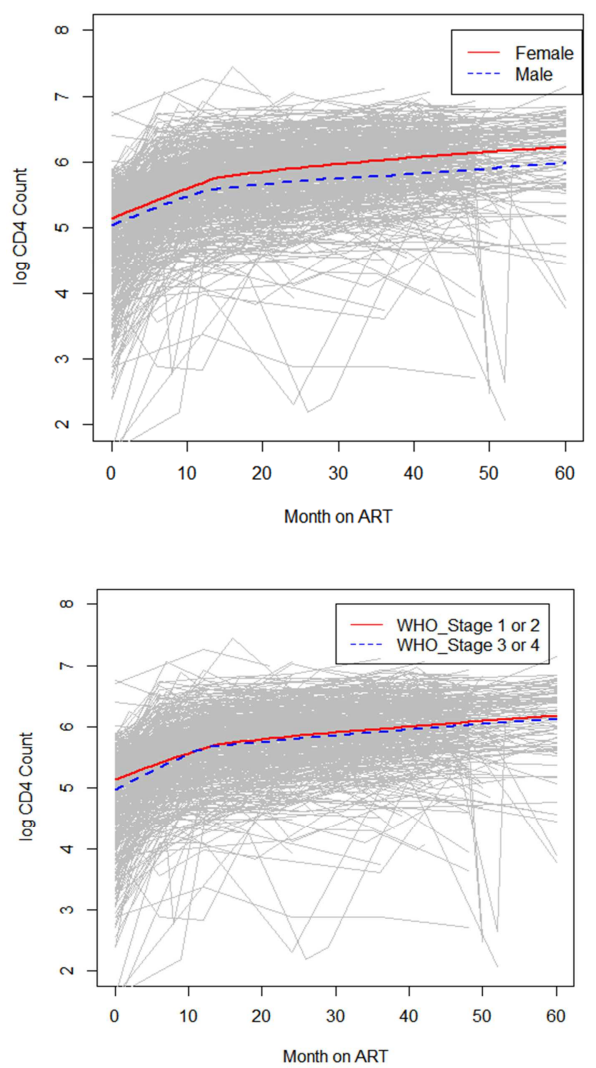
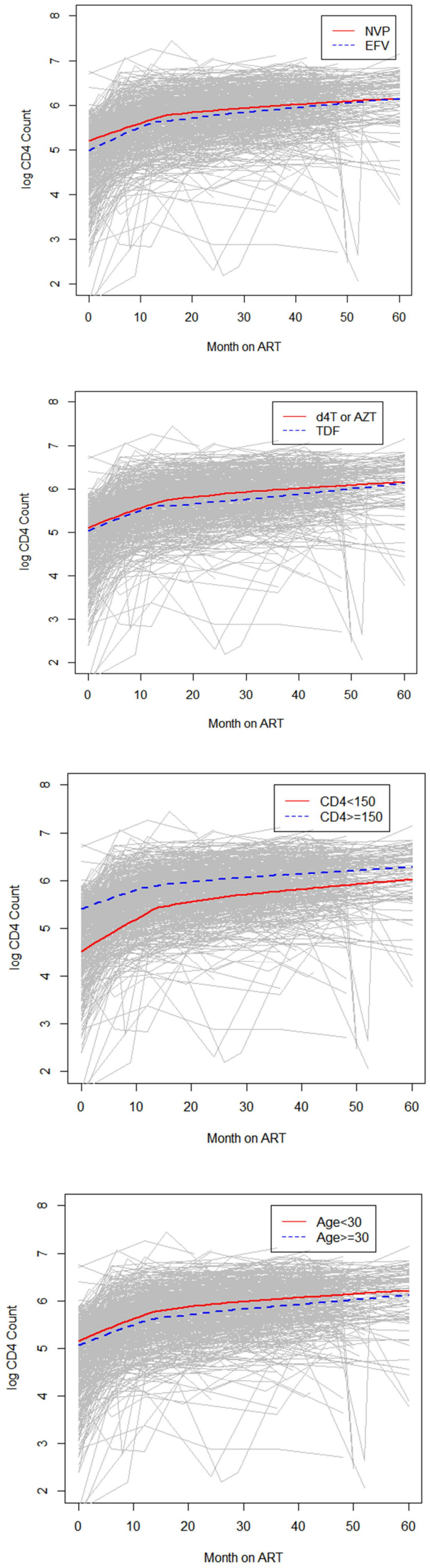

Figure 1. Individual CD4 profile and smoothed CD4 count over time on ART by sex, clinical stage, ART regimen, CD4 count at ART initiation, and age at ART initiation. 
In order to evaluate the association between independent variables and CD4 response over time on ART the proposed liner mixed model (1) is fitted by using the lme procedure available in $\mathrm{R}$ package nlme. The results of the final model are shown in Table 2. Being under HAART for long period, gender, clinical stage and baseline CD4 count are associated with CD4 profile over time. For every additional month on ART you can expect CD4 count to increase by an average of 5.5 in the log scale or $220 \mathrm{CD} 4$ count per $\mathrm{mm}^{3}$ and males have observed to have a lower CD4 increment profile as compared to females. Being in WHO stage 3 or 4 at the start of therapy was found also to have a negative effect on the CD4 increment over time. No difference in CD4 count over time was observed according to initial HAART treatment.

Table 2. Parameter estimates.

\begin{tabular}{llll}
\hline & Estimate & Std.Error & p-value \\
\hline Intercept & 4.739114 & 0.045131 & $0.000^{*}$ \\
Month on Art & 0.055092 & 0.001794 & $0.000^{*}$ \\
Sex_male & -0.095 & 0.038646 & $0.014^{*}$ \\
WHO_Stage 3 or 4 & -0.07765 & 0.03783 & $0.041^{*}$ \\
Baseline CD4 $>150$ & 0.688497 & 0.03591 & $0.000^{*}$ \\
NNRTI EFV & -0.03606 & 0.041227 & 0.3821 \\
NRTI TDF & -0.07245 & 0.048487 & 0.1358 \\
$\delta_{b_{0, s}}^{2}$ & $1.89 \mathrm{E}-02$ & & \\
$\delta_{b_{1, S}}^{2}$ & $5.09 \mathrm{E}-06$ & & \\
$\rho_{b_{0, s}, b_{1, S}}$ & 0.005 & & \\
$\delta_{b_{0, I(s)}}^{2}$ & 0.324043 & & \\
$\delta_{b_{1, I(s)}}^{2}$ & 0.011008 & & \\
$\rho_{b_{0, I(s),}, b_{1, I(s)}}$ & -0.176 & & \\
$\delta^{2}$ & 0.425296 & & \\
\hline
\end{tabular}

\section{Discussion}

The study combined data from two health centers from resource-limited settings in South Western Ethiopia to assess CD4 trajectories after ART initiation at sites engaged in the scale up of HIV care and treatment. A total of four hundred seventy six patients, 240 from Mizan -Tepi Teaching Hospital and 236 from Tepi General Hospital, were followed for a maximum of three years. The data reveal robust CD4 responses to ART that are continual over years. The study results are thus encouraging regarding the long-term effectiveness of ART in resource-limited settings, but they naturally are only applicable to those who are able to remain on ART for extended periods.

In this study, we found several factors associated with CD4 count increment over time. Being on ART for extended period was the main factor associated with CD4 count profile over time. Males were observed to have a lower gain in CD4 count over time as compared to females. Patients that start ART at late stage (Stage 3 or 4 ) have a lower CD4 count gain over time as compared to patients that start ART at early stage. Starting ART while having a CD4 count greater than 150 was also found to have a positive effect.

The findings on long-term CD4 response appear to be consistent with those of other long-term investigations in [2, 9] which examined immunologic response up to 3, 5 and 6 years after ART initiation, respectively.
The study found an intriguing finding, differences in CD4 trajectories by sex, where females had higher baseline CD4 counts which is similar with some other studies [3,5], but we did not find any justification for this and additional research is required.

\section{Strengths and Limitations}

Even though, perfect eradication of HIV from an individual is not currently possible, we can delay the progression of disease by using HAART which provide protection against the development of HIV-related complications by a long-term increase in CD4 cells. Therefore this study helps to understand the importance of attending HAART program by showing how much CD4 cells increase over time so that the patient's functional status and disease protective ability also increase or simply how much it delay disease progression. The data used for this study is very used full, timely, and appropriate statistical methods were used. Thus, the current study is generalizable and it is regarded as the strength of the study. Where are some exclude a substantial number of patients due to lack of follow-up CD4 counts and these patients may differ systematically from those who were included in the analysis. Our analysis will thus probably overestimate or underestimate the true impact of ART on CD4 counts in all patients initiating ART.

\section{Conclusion}

The study demonstrates robust CD4 response to ART among patients in multiple treatment programs in resource-limited settings. The response appears to be sustained among those remaining in programs for up to 3 years. The results thus support the notion that a programmatic, public health approach to ART in resource-limited settings using a limited number of NNRTI based drugs can result in sustained immunologic outcomes that are comparable to industrialized countries.

\section{Recommendation}

Because of the limited number and follow-up of patients who were failing HAART, this analysis is currently underpowered. Further analyses will be considered with longer duration of follow-up.

\section{Abbreviations}

\author{
AIDS: Acquired Immune Deficiency Syndrome \\ ART: Antiretroviral Treatment \\ ARV: Antiretroviral \\ CD4: Cluster of differentiation 4 \\ HAART: HIV/AIDS Anti Retroviral Treatment \\ WHO: World Health Organization
}

\section{Authors Contributions}

Both are responsible for the conception, data analysis, report, preparation of the draft manuscript, and interpretation of the results with crosschecking. 


\section{Ethics of Approval and Consent to Participate}

Ethical clearance was obtained from Mizan-Tepi University Teaching Hospital and Tepi General Hospital of South Western Ethiopia. Careful recruitment and training for data collectors was undertaken. To maintain the confidentiality, the data collectors (two nurses and one data clerk) was extracted the necessary data from the patient baseline and follow up card. The data obtained by checklist was collected by the principal investigator on daily basis.

\section{Funding}

The author disclosed receipt of the following financial support for the research, authorship, and/or publication of this article: The study was fully funded by Mizan-Tepi University. However, they have no role in the design of the study and collection, analysis, and interpretation of data and in writing the manuscript.

\section{Acknowledgements}

The study would like to greatly acknowledge Mizan-Tepi University Teaching Hospital and Tepi General Hospital HIV Outpatient Clinic for allowing us to use the HAART data. In addition, all who contributed for data collection are duly acknowledged.

\section{References}

[1] M. R. Loutfy, L. Sherr, U. Sonnenberg-schwan, S. L. Walmsley, and M. Johnson, "Review article Caring for women living with HIV : gaps in the evidence," pp. 1-14, 2013.

[2] M. E. Arrivé, R. Becquet, F. D. Coordinator, and V. Leroy, "HIV Care \& PMTCT in Resource-Limited Settings Available on line prepared by the Bordeaux Working Group," vol. 2, no. 8, pp. 39-50, 2006.

[3] B. S. C. Uzochukwu, O. E. Onwujekwe, A. C. Onoka, C. Okoli, N. P. Uguru, and O. I. Chukwuogo, "Determinants of non-adherence to subsidized anti-retroviral treatment in southeast Nigeria," no. March, pp. 189-196, 2009, doi: 10.1093/heapol/czp006.

[4] I. Zulu et al., "Rapid Scale-up of Antiretroviral Therapy at Primary Care Sites in Zambia Feasibility and Early Outcomes," vol. 296, no. 7, 2006.

[5] Y. Madec, D. Laureillard, and L. Pinoges, "Response to highly active antiretroviral therapy among severely immunocompromised HIV-infected patients in Cambodia. Authors Response to highly active antiretroviral therapy among severely immuno-compromised $\mathrm{HIV}$-infected patients in Cambodia," 2020.

[6] T. Opportunistic, I. Project, and O. Hiv, "CD4 Cell Count and the Risk of AIDS or Death in HIV- Infected Adults on Combination Antiretroviral Therapy with a Suppressed Viral Load : A Longitudinal Cohort Study from COHERE," vol. 9, no. 3, 2012, doi: 10.1371/journal.pmed.1001194.
[7] S. Du Mortier, S. Mukangu, C. Sagna, L. Nyffenegger, and S. A. Perone, "A decade of an HIV workplace programme in armed conflict zones; a social responsibility response of the International Committee of the Red Cross," J. Occup. Med. Toxicol., pp. 1-10, 2016, doi: 10.1186/s12995-016-0119-4.

[8] W. H. O. E. Region, HIV / AIDS TREATMENT AND CARE Clinical protocols for the WHO European Region HIV / AIDS TREATMENT AND CARE Clinical protocols for the WHO European Region.

[9] O. J. Daniel, O. Ot, O. A. Ogundahunsi, and S. Fagbenro, "Default from Anti-Retroviral Treatment Programme in Sagamu, Nigeria," vol. 11, no. May, pp. 221-224, 2008.

[10] S. Mcgee, J. D. Kuruc, J. L. Lennox, and D. M. Margolis, "NIH Public Access," vol. 62, no. 5, pp. 505-508, 2014, doi: 10.1097/QAI.0b013e318285cd33. Antiretroviral.

[11] O. Erhabor, O. A. Ejele, and C. A. Nwauche, "stavudine, lamivudine and nevirapine on the CD4 lymphocyte count of HIV-infected Africans : the Nigerian experience," vol. 1, p. 39, 2009, doi: 10.1186/1742-4690-6-S2-P66.

[12] J. Zhou, T. Sirisanthana, S. Kiertiburanakul, Y. A. Chen, N. Han, and P. L. Lim, "Trends in CD4 counts in HIV-infected patients with HIV viral load monitoring while on combination antiretroviral treatment: results from The TREAT Asia HIV Observational Database," BMC Infect. Dis., vol. 10, no. 1, p. 361, 2010, doi: 10.1186/1471-2334-10-361.

[13] A.. Bowman, F. D. and Manatunga, "A joint model for longitudinal data Profiles and associated event risks with application to a depression study," Appl. Stat., vol. 2, no. 54, pp. 301-316, 2005.

[14] L. Liang, K. and Zeger, Longitudinal data analysis for discrete and continuous outcomes. 1982.

\section{Biography}

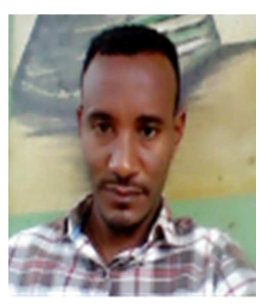

Solomon Abebaw Andargie I have graduated with BSc degree in Statistics from University of Gondar. Immediately after my graduation, I joined Mizan Tepi University as a graduate assistant and served there for two years as an assistant lecturer. Then I have joined Jimma University to study Masters of Science in Biostatistics and graduated in 2016. Now, I am lecturer at Mizan-Tepi University, College of Natural and Computational science, Department of statistics.

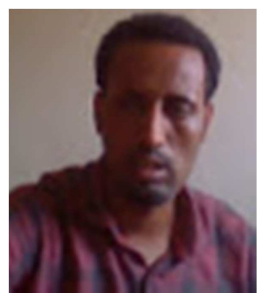

Assaye Belay Gelaw was graduated B.Sc degree in Statistics from Wollega University in 2010 G.c. Ministry of higher education has assigned me in Mizan-Tepi University as assistant instructor I and II. After two years' service of teaching, I have joined to Jimma University to study M.Sc. in Biostatistics and graduated in 2015 G.c. Now, I am lecturer at MizanTepi University College of Natural and Computational Science, Department of Statistics. 